\title{
Videojuegos educativos y pensamiento científico: análisis a partir de los componentes cognitivos, metacognitivos y motivacionales ${ }^{1}$
}

\section{Jairo Andrés Montes González}

orcid.org/oooo-0003-1778-1096

Pontificia Universidad Javeriana de

Cali, Colombia

jamontes@javerianacali.edu.co

\section{David S. Baldeón Padilla}

orcid.org/oooo-0oo1-9540-2528

Pontificia Universidad Javeriana de

Cali, Colombia

david.baldeon@javeriancali.edu.co
Solanlly Ochoa-Angrino

orcid.org/0000-0002-7043-2808 Pontificia Universidad Javeriana de Cali, Colombia

sochoa@javerianacali.edu.co

\section{Mariana Bonilla Sáenz}

orcid.org/0000-0003-4238-050X Pontificia Universidad Javeriana de Cali, Colombia mbonillag6@javerianacali.edu.co

\section{Resumen}

Se presenta una revisión de literatura con el objetivo de identificar las diferentes posturas en investigación sobre el uso de los videojuegos en contextos educativos. Estas posturas fueron diferenciadas a partir de dos categorizaciones. En primer lugar, se categorizaron aspectos privilegiados por las investigaciones para el favorecimiento del desarrollo del pensamiento científico, como aspectos cognitivos, metacognitivos y/o motivacionales. En segundo lugar, se clasificó el tipo de representación que las investigaciones asumian al integrar videojuegos en la educación. Esta clasificación derivó en el planteamiento de dos niveles de representación. El nivel de integración, que hace referencia a la concepción del uso de videojuegos a partir de su potencial para presentar contenidos, transmitir y comunicar información, y el nivel de reorientación, que se refiere a una concepción donde el videojuego no es el foco, sino su potencial para fomentar habilidades de alto orden, construir conocimiento y generar interacciones en un contexto educativo. Como conclusión, se hace referencia a

$1 \quad$ El presente artículo es producto del proyecto de investigación "Pensamiento científico y videojuegos: Aproximación microgenética a la experimentación, la motivación y el aprendizaje colaborativo" identificado con el código 020100548 y desarrollado por la Pontificia Universidad Javeriana Cali.

Recepción: 19/04/2018 | Envío a pares: 10/08/2018 | Aceptación por pares: 24/og/2018 | Aprobación: 11/11/2018 DOI: 10.5294/edu.2018.21.3.2

Para citar este artículo / To reference this article / Para citar este artigo

Montes-González, J. A., Ochoa-Angrino, S., Baldeón-Padilla, D. S. y Bonilla-Sáenz, M. (2018). Videojuegos educativos y pensamiento científico: análisis a partir de los componentes cognitivos, metacognitivos y motivacionales. Educación y Educadores, 21(3), 388-408. DOI: 10.5294/edu.2018.21.3.2 
la importancia de tener en cuenta bajo qué representación, condiciones y características se hace uso de un videojuego en contextos educativos, puesto que eso podrá favorecer o no la potencialidad del uso de un videojuego como herramienta cultural en la educación.

\title{
Palabras clave (fuente: tesauro de la Unesco)
}

Metacognición; motivación; resolución de problemas; tecnología educacional; videojuego.

\section{Educational Videogames and Scientific Thinking: Analysis from the Cognitive, Metacognitive and Motivational Components}

\begin{abstract}
A literature review is presented to identify diverse research positions on the use of videogames in educational contexts. These positions were differentiated based on two categorizations. Firstly, aspects privileged by research for favoring scientific thinking development, such as cognitive, metacognitive and/or motivational components, were categorized. Secondly, the type of representation that research assumed when introducing videogames in education was classified. This classification derived in the proposal of two levels of representation: the level of integration, which refers to the conception of the use of videogames for their potential to present contents, transmit and communicate information; and the level of reorientation, which refers to a conception where the videogame is not the focus, but its potential to promote highorder skills, build knowledge and create interactions in an educational context. It is concluded that it is relevant to define under what representation, conditions and characteristics a videogame is used in educational contexts since this may or may not bolster the potential of this resource as a cultural tool in education.
\end{abstract}

\section{Keywords (Source: Unesco thesaurus)}

Metacognition; motivation; problem solving; educational technology; video games. 


\section{Videogames educativos e pensamento científico: análise a partir dos componentes cognitivos, metacognitivos e motivacionais}

\section{Resumo}

Apresenta-se uma revisão de literatura com o objetivo de identificar as diferentes posturas em pesquisa sobre o uso dos videogames em contextos educativos. Essas posturas foram diferenciadas a partir de duas categorizações. Em primeiro lugar, foram categorizados os aspectos privilegiados pelas pesquisas para o favorecimento do desenvolvimento do pensamento científico, como os cognitivos, metacognitivos el ou emocionais. Em segundo lugar, classificou-se o tipo de representação que as pesquisas assumiam ao integrar videogames na educação. Essa classificação resultou em uma abordagem de dois niveis de representação: o nivel de integração, que se refere à concepção do uso de videogames a partir de seu potencial para apresentar conteúdos, transmitir e comunicar informação; e o nível de reorientação, que se refere a uma concepção na qual o videogame não é o foco, mas sim seu potencial para fomentar habilidades de alta ordem, construir conhecimento e gerar interações em um contexto educativo. Conclui-se que é importante decidir sob quais representações, condições e características se usa um videogame em contextos educativos, visto que isso poderá favorecer ou não o potencial desse recurso como ferramenta cultural na educação.

\section{Palavras-chave (Fonte: tesauro da Unesco)}

Metacognição; motivação; resolução de problemas; tecnologia educacional; videogame. 
Cuando un niño de 9 años se sienta a jugar por primera vez Spore (un juego de simulación de vida y estrategia) empieza por tratar de entender cómo se juega, cómo usar los controles y qué objetivo debe alcanzar para poder ganar. En primera instancia explora las características del videojuego y ensaya la manera de jugarlo, basándose en hipótesis que formula a partir de su experiencia con otros videojuegos. Parte de sus hipótesis son correctas, pero en otros casos debe pensar en nuevos patrones de juego que se acoplen a las exigencias del nuevo videojuego. De esta manera, descubre que su objetivo como jugador es desarrollar un organismo que evolucione desde la vida unicelular hasta la vida en civilización.

Diseñando su primer organismo, empieza a encontrar obstáculos que no le permiten avanzar con facilidad en el juego (especies rivales, depredadores, dificultades para conseguir alimento, entre otras). El niño debe entonces manipular las características del organismo que ha creado, en términos de sus estrategias de locomoción, alimentación, habilidades sociales y uso de herramientas. Durante esta manipulación encuentra resultados, a veces buenos y a veces no tanto, para superar las metas a corto plazo que plantea el juego y avanzar de nivel. Las metas van cambiando conforme el juego avanza; por ende, las estrategias también deben transformarse de manera adaptiva. Esta interacción dinámica entre las condiciones cambiantes de juego y las respuestas adaptativas del niño se dan hasta que tiene éxito en el vídeo juego.

Este tipo de dinámica compleja de comprensión es semejante a la que se encuentra cuando el niño se enfrenta a un problema de la vida real que requiere la puesta en marcha del pensamiento científico. En este sentido, el niño es capaz de identificar variables, predecir el resultado de interacciones entre variables y reconocer entre las variables causales y las que no lo son. Además, le permite dar sentido a la información disponible y en algunos casos hacer comparaciones controladas entre variables para inferir conclusiones (Montes, Van Dijk, Puche y Van Geert, 2017).
De manera específica, el pensamiento científico da cuenta del razonamiento y las habilidades de resolución de problemas puestas en marcha al generar, poner a prueba y evaluar teorías que permitan explicar un fenómeno (Zimmerman y Schunk, 2012). Este tipo de razonamiento implica actividades cognitivas complejas como la formulación de hipótesis, la postulación de causas y sus relaciones con los efectos que acarrean, la coordinación de teoría y evidencia y el control de variables (Kuhn, 2010; Kuhn y Pease, 2008, Puche y Ordóñez, 2003).

Teniendo en cuenta la complejidad de habilidades involucradas en el pensamiento científico, es necesario plantear que estas habilidades no se desarrollan de manera espontánea y deben ser mediadas por la educación en ciencias y por las herramientas culturales (Morris, Croker, Zimmerman, Gill y Romig, 2013). Una de estas posibles herramientas culturales son los videojuegos.

En este sentido, cabe preguntar: ¿qué características tienen los videojuegos como situaciones de resolución de problemas que los hacen particulares y los diferencian de otros tipos de herramientas culturales?, ¿qué aspectos de los videojuegos pueden favorecer el desarrollo del pensamiento científico? y ¿al diseñar un videojuego o investigar el potencial de los videojuegos en educación en ciencia, tecnología, ingeniería y matemáticas (STEM, en inglés), en cuáles variables se debe centrar la atención? Estas preguntas no son fáciles de responder e implican un análisis profundo de las habilidades cognitivas, metacognitivas y motivacionales que pueden emerger en el momento en el que un niño o adolescente entra en interacción con un videojuego educativo.

Estas preguntas son relevantes teniendo en cuenta el auge de la literatura en el área y la creciente inversión en el diseño de videojuegos educativos, áreas en las cuales muchas veces se asume que su sola presencia trae beneficios en la calidad educativa. En particular, esta revisión parte de la idea según la cual es necesario comprender el carácter complejo 
de la relación entre el jugador (momento del desarrollo, nivel de conocimiento previo del dominio del juego, intereses y motivaciones), las características de los videojuegos con los que interactúa (situación o problema que recrea el videojuego, narrativa, niveles de dificultad), las características del docente, en particular de sus prácticas pedagógicas (es decir, usos concretos de los videojuegos en el salón de clase) y el contenido disciplinar que se aborda a través del juego (videojuegos en ciencias naturales, en ciencias sociales, etc.), debido a que el pensamiento científico emerge como un producto de la interacción entre estos factores (Kuhn, 2011; Morris et al., 2013).

En este orden de ideas, es posible identificar que el tipo de representación que tienen los investigadores acerca de los videojuegos como herramienta educativa producirá variación en lo que ellos consideran potencialmente relevante para para favorecer el pensamiento científico. En este caso, al realizar una revisión sistemática de las investigaciones, y la complejidad de las representaciones que subyacen a ellas, es posible encontrar principios aplicables al diseño, implementación y evaluación de la incorporación de videojuegos como herramienta cultural a contextos educativos.

\section{Niveles representacionales en el uso de videojuegos educativos: componentes cognitivos, metacognitivos y motivacionales}

A partir de la presente revisión se han podido identificar dos niveles de representación acerca del potencial educativo de los videojuegos. El primero se denominará como integración y el segundo como reorientación. Estos niveles surgen de una adaptación de modelos de integración de tecnologías de la información y la comunicación (TIC) a contextos educativos (Hooper y Rieber, 1995; Montes y Ochoa, 2006; Valencia et al., 2017). En términos generales, el nivel de integración da cuenta del uso de videojuegos a partir de su potencial para presentar contenidos, transmitir y comunicar información. En este senti- do, se privilegian las posibilidades que tienen estas herramientas para demostrar, describir, ejemplificar y clarificar información, haciendo que el aprendiz/ jugador se apropie de dicha información de manera más amena, pertinente y cercana a sus gustos.

Por otro lado, el nivel de reorientación implica un uso más reflexivo de las TIC, en donde el foco no se encuentra en el videojuego mismo, sino en su potencial para fomentar habilidades de alto orden, construir conocimiento y generar interacciones que sin su uso sería poco probable que ocurriesen en un contexto educativo. Así mismo, se hace uso de algunas de sus características particulares como instrumento de mediación: interactividad, formalismo, dinamismo, multimedia e hipermedia (Martí, 2003; Valencia et al., 2017).

A continuación, se caracterizarán los dos niveles partiendo de cómo pueden favorecer los aspectos cognitivos, metacognitivos y motivacionales de un aprendiz al hacer uso de un videojuego educativo (Figura 1) (Morris et al., 2013). Los aspectos cognitivos son entendidos como todos aquellos procesos perceptuales y de pensamiento que se ven involucrados en un ambiente donde se hace uso de videojuegos educativos. Los aspectos metacognitivos son tenidos en cuenta como un importante componente a la hora de hacer uso de videojuegos educativos centrándose en el monitoreo, control y planificación del conocimiento y habilidades propias. Este monitoreo será esencial para lograr la construcción de conocimiento y el desarrollo de habilidades en el jugador/aprendiz. Por último, los aspectos motivacionales son quizás el factor más importante tenido en cuenta para hacer uso de videojuegos en la educación, por la facilidad que tiene un videojuego para lograr que emerjan experiencias óptimas como el fluir (flow) y el compromiso (Hamari et al., 2016) y se mantengan en el tiempo, a pesar de los obstáculos que el jugador/aprendiz encuentre en su camino (Morris et al., 2013). 


\section{Figura 1. Niveles de representación del potencial educativo de los videojuegos}

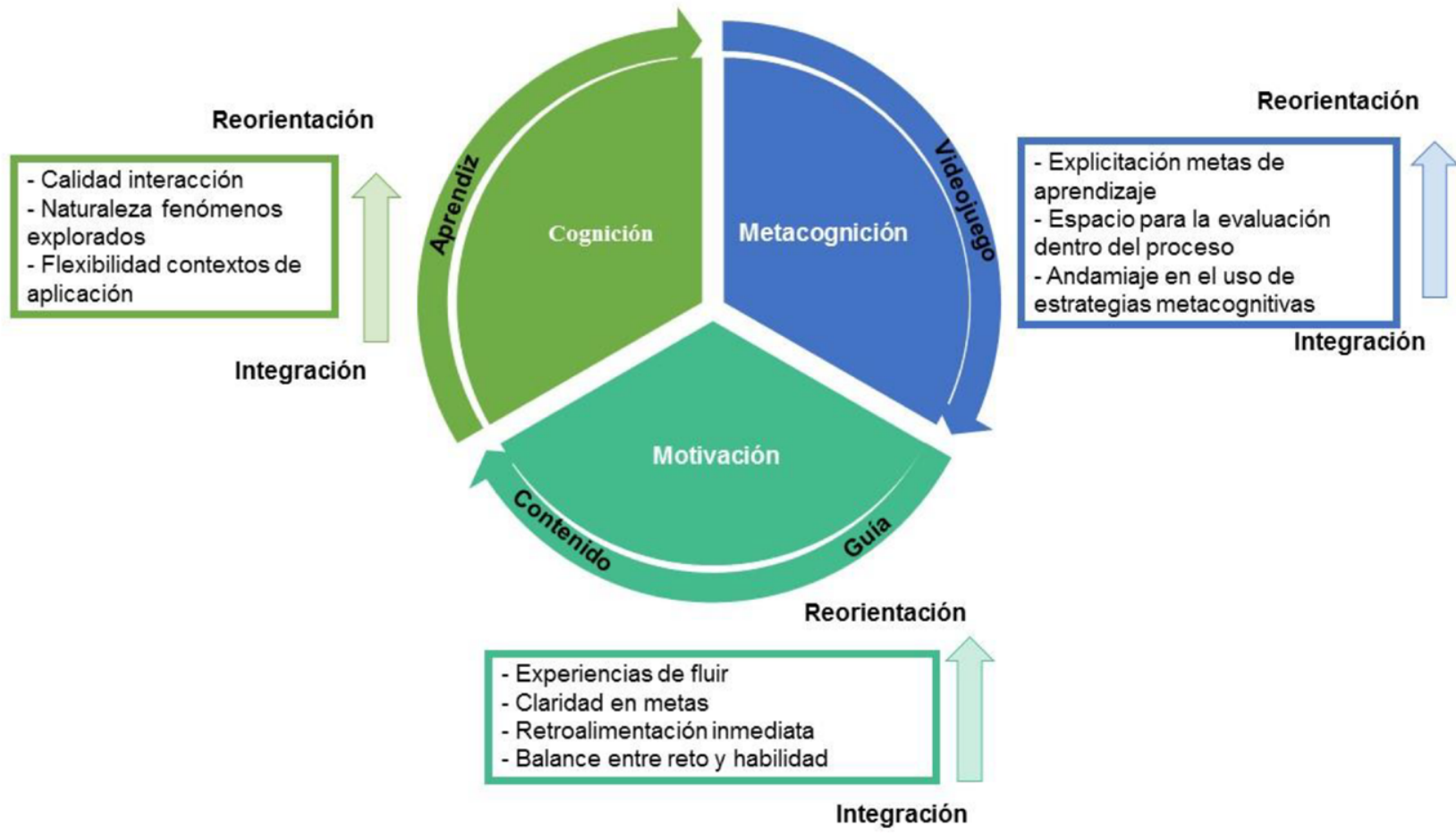

Fuente: elaboración propia.

\section{Componentes cognitivos}

En cuanto a los aspectos cognitivos, tres características serán las abordadas desde los niveles de integración y reorientación. Estas características permiten que un videojuego educativo logre el objetivo de aprendizaje deseado, la construcción de conocimiento y/o el desarrollo de habilidades. Estas características son: calidad de la interacción, naturaleza de los fenómenos explorados y flexibilidad de los contextos de aplicación.

En primer lugar, la calidad de la interacción da cuenta de la representación de jugador de la que se parte al diseñar el videojuego, de su intencionalidad educativa y de la forma en la que se estructura la interacción entre estos. Por otra parte, la naturaleza de los fenómenos explorados se centra en la complejidad de las representaciones abordadas en los contenidos del videojuego. Finalmente, la flexibilidad de los contextos de aplicación describe el nivel de explicitación de la relación entre los contextos donde se espera que el estudiante aplique lo aprendido, así como del nivel de organización predefinida y la flexibilidad de los caminos para alcanzar las metas planteadas por el videojuego.

\section{Calidad de interacción jugador-videojuego}

En cuanto a la calidad de la interacción, el nivel de integración se basa en el favorecimiento de la transmisión de información, implicando elementos tanto de forma como de contenido del videojuego. Los elementos de forma hacen referencia al material perceptual que el videojuego educativo le provee al aprendiz. Por otra parte, el contenido del videojuego hace referencia a la narrativa, conceptos y contextos que el videojuego presenta a lo largo de su desarrollo. Estos elementos se articulan con el objetivo de proveer información relevante al aprendizaje de contenidos por parte del jugador. 
Un ejemplo en el cual se privilegian estas características es el estudio de Quintanal (2016) donde se buscaba que estudiantes de bachillerato hicieran uso de minijuegos web para el aprendizaje de física y química. Estos minijuegos fueron seleccionados por profesores que tuvieron en cuenta que los aspectos de forma y contenido se relacionaran con el área de conocimiento que buscaban fortalecer. Los criterios para incluir los juegos en la lista eran: que su forma fuera adecuada (calidad de imagen, sonido, interfaz), que correspondiera a la edad del estudiante y que tuviera un nivel de lenguaje adecuado. Los resultados indicaron que los estudiantes lograron los objetivos de la actividad de aprendizaje, asumiendo este logro por el alza en niveles de motivación, autoconfianza y autonomía.

Pese a que el uso de estos videojuegos puede resultar beneficioso para los procesos de aprendizaje de los estudiantes, los criterios de selección de los investigadores estuvieron centrados en que la información presentada fuera comprensible y permitiera asociaciones con los temas de clase. No obstante, el videojuego puede presentar un material con contenido relevante y no necesariamente tener una intencionalidad dirigida a permitir interacciones que favorezcan la construcción de conocimiento de alto orden.

Es por esto que resulta importante conectar los contenidos educativos con videojuegos. Sin embargo, además de comunicar estos contenidos, desde una perspectiva de reorientación, se busca que el estudiante tenga una participación activa en problemas que impliquen el uso de estos contenidos y se favorezca la transformación de su conocimiento previo.

Desde esta perspectiva, no debe confundirse la resolución de problemas con el aprendizaje mecánico a través de la resolución de ejercicios. De hecho, es bastante común la estructuración de los juegos con base en la presentación de ejercicios de repetición mecánica, tales como operaciones matemáticas aisladas o tareas de memoria para recordar hechos y datos, desligadas de las otras acciones de juego.
Un ejemplo en esta dirección es el Math Blaster (Holbert y Wilensky, 2014). En este videojuego, se deben resolver problemas matemáticos en el contexto del espacio exterior. No obstante, aunque estos problemas matemáticos estén en medio del juego, estos solo se resuelven para que el jugador pueda acceder a una mejora, lo que configura una estructura de recompensa (poder jugar) al resolver el problema aritmético planteado. Estos problemas matemáticos se plantean como operaciones formales aisladas de conceptos de aplicación, de contextos significativos para el estudiante e incluso de la narrativa del videojuego. En conclusión, cualquier videojuego siempre implicará resolver problemas, solo que a veces los problemas poco tienen que ver con el contenido (Rice, 2007).

En contraste, un ejemplo del manejo del contenido en el nivel de reorientación se encuentra el estudio de Clark et al. (2011). En el videojuego que analizan, la forma se basaba en figuras geométricas básicas en tercera dimensión y el contenido se presentaba desde la narrativa, como un conjunto de misiones necesarias para cumplir el objetivo de salvar a la galaxia. A partir de los conceptos, se debía hacer uso de concepciones físicas como velocidad, tiempo, aceleración y colisiones para poder cumplir las misiones. Por otra parte, respecto del contexto, se presentaba el videojuego en un ambiente espacial donde se hacía uso de elementos como estrellas, naves, satélites, entre otros. De esta manera, se realiza el anclaje necesario entre el objetivo educativo del videojuego y la forma-contenido del mismo, evitando caer en modelos donde la práctica mecánica es el móvil usado para lograr el aprendizaje (Shaffer, Squire, Halverson y Gee, 2005).

Además, en el nivel de reorientación, también se tiene en cuenta el favorecimiento de una construcción dinámica del conocimiento, que promueva variar las estrategias cognitivas (Morris et al., 2013), formular nuevas hipótesis y ajustar nueva evidencia a las mismas. Este cambio constante se verá reflejado en la aparición de nuevas metas a partir del 
desempeño del jugador/aprendiz mientras juega. Además, cuando emergen estas nuevas metas, los niveles de demanda estarán continuamente ajustándose a las habilidades, que estarán igualmente ajustándose con las demandas de las metas emergentes, lo que le da un carácter bidireccional y recursivo a esta relación. De esta forma, se obtiene una mejora en la calidad de la interacción, al encontrar mecanismos de adaptación dinámicos tanto en el videojuego como en el jugador/aprendiz.

En este sentido, Schulze et al. (2015) fomentan la emergencia de nuevas metas en el diseño de videojuegos educativos, le aportan un carácter dinámico, que se aleja de la linealidad en su despliegue, y favorecen el aprendizaje y desarrollo de habilidades cognitivas complejas. Así, para que el jugador/ aprendiz logre este aprendizaje y desarrollo de habilidades que no emergen de manera espontánea (Anderson et al., 2009; Morris et al., 2013), propias del pensamiento científico, es necesario que las nuevas metas emergentes impliquen la resolución de problemas que tengan una complejidad creciente. De esta forma, la adaptación constante será necesaria y favorecerá procesos de andamiaje donde el jugador/aprendiz logrará niveles superiores de habilidad y conocimiento a medida que avanza el videojuego.

De igual manera, la variabilidad en la dinámica de juego se verá favorecida por esta constante adaptación en la demanda del videojuego y las habilidades del jugador/aprendiz. Variabilidad que hace referencia a los diversos cambios que pueden ocurrir en el desarrollo del videojuego y en las constantes transformaciones de las condiciones del mismo (Halpern et al., 2012).

Ejemplificando lo anterior, se encuentra el estudio realizado por Schulze et al. (2015), donde se tenía en cuenta esta variabilidad y emergencia de nuevas metas en la dinámica del videojuego. En el juego, estudiantes de bachillerato y universidad de- bían manejar de la mejor forma posible los recursos humanos y naturales con el objetivo de aumentar sus recursos financieros. Para lograr el éxito debían tener en cuenta múltiples variables que podían cambiar de un momento a otro. Entre estas variables se mencionan economía, la salud, estaciones del año, clima, entre otras. De esta forma, la interacción entre el videojuego y el jugador/aprendiz se ve enriquecida por los nuevos elementos que provee el videojuego y la adaptación de habilidades del jugador/aprendiz a estas nuevas demandas.

\section{Naturaleza de los fenómenos que se exploran}

La segunda característica abordada en los aspectos cognitivos es la naturaleza de los fenómenos explorados. Esta, en el nivel de integración, se encuentra dirigida al entendimiento de fenómenos simples. Este tipo de fenómenos parten de relaciones monocausales entre elementos, las cuales generalmente se traducen en la forma de acciones y respuestas, donde las acciones realizadas por el jugador generan un único efecto en la situación de juego, debido a que no se contemplan fenómenos multivariables y, por ende, las acciones del jugador/ aprendiz se limitan a ser correctas o incorrectas. En este sentido, solo cuando la acción realizada es coherente con la estructura predefinida por el videojuego, se alcanza la meta. Este proceso simplificado limita el potencial educativo de un videojuego para el aprendizaje de conceptos y fenómenos complejos, pues le presenta al jugador/aprendiz un campo de acción con pocas posibilidades (Miller, Chang, Wang, Beier y Klisch, 2011; Yang, 2012).

Por ejemplo, el estudio de Hou y Li (2014) buscó evaluar la efectividad del uso de videojuegos en el aprendizaje de conceptos y ensamblaje de computación en estudiantes universitarios. La dinámica del videojuego se basaba en cumplir procedimientos fijos, donde las posibilidades de respuesta eran pocas, lo que lo convierte en un fenómeno excesivamente simplificado. Esto lleva al aprendiz a usar estrategias simples como el ensayo y error, 
estrategias que son valiosas para el desarrollo de habilidades cognitivas, pero que, debido a su linealidad, limitan el potencial que puede ofrecer un videojuego para el aprendizaje.

Por otra parte, cuando la comprensión de fenómenos explorados se ubica en el nivel de reorientación, la comprensión estará encaminada a fenómenos de carácter complejo y dinámico. Lo anterior implica que el videojuego se basará en la presentación de problemas con una estructura variable (Halpern et al., 2012) y las múltiples soluciones que pueden surgir de las acciones del jugador/ aprendiz (Annetta, 2008; Lester et al., 2014). Esta dinámica favorecerá el uso y desarrollo de habilidades propias del pensamiento científico e implicará la adaptación para poder responder a estas condiciones cambiantes. El aprendiz deberá analizar la información recibida, construir hipótesis, pensar soluciones alternativas y hacer predicciones. Todo esto haciendo uso de diversos argumentos, conclusiones y explicaciones.

Por ejemplo, Squire (2008) presenta el videojuego Civilization como una herramienta potencial para construir nuevos conocimientos sobre historia desarrollando habilidades de pensamiento científico. Ubicación geográfica, civilizaciones, recursos, acuerdos de mercado, oportunidades de expansión y tecnologías son elementos en constante movimiento que el jugador/aprendiz debe tener en cuenta para cumplir las metas y avanzar. La presencia de todos estos elementos y su variabilidad les da un carácter complejo a los fenómenos que se desarrollan en el videojuego. Esta complejidad favorecerá el uso y desarrollo de habilidades de alto orden en situaciones como el análisis e interpretación de la información recibida sobre cada época, el planteamiento y comprobación de hipótesis sobre cuáles son los mejores caminos a seguir para el avance de su civilización, conclusiones y posibles explicaciones sobre modelos económicos, políticos y sociales más o menos favorables a su civilización.

\section{Flexibilidad de los contextos de aplicación}

La última característica de los aspectos cognitivos es la flexibilidad de los contextos de aplicación. En el nivel de integración, se parte de la idea según la cual basta con tomar un aspecto de los contextos reales para simularlo en el juego y así desarrollar una habilidad o un conocimiento. Esta simulación se realiza a partir de problemas bien definidos, una estructura de problemas que, en su organización y contenido, proveen todo el material e información necesarios para que el aprendiz/jugador pueda resolver los problemas. En este sentido, los posibles caminos para llegar a la solución están predefinidos y el número de reglas que rigen el problema son limitados. Este tipo de problemas son los que se encuentran comúnmente en actividades de aprendizaje de la educación formal (Jonassen, 2011). En consecuencia, se asume que los estudiantes aplicarán a contextos reales los conocimientos y habilidades específicas desarrolladas en el juego (Anderson y Barnnet, 2011; Barab y Dede, 2007; Cheng, Su, Huang y Chen, 2014). No obstante, los elementos que se toman de la realidad están "atomizados" y no toman en cuenta la complejidad de los fenómenos explorados. En estos casos, no se permite que el estudiante explore y encuentre los principios subyacentes a los problemas a resolver.

Un ejemplo de esto lo da el estudio de Barzilai y Blau (2014) donde se buscó identificar cómo un videojuego podía favorecer el aprendizaje de problemas matemáticos financieros en estudiantes de secundaria. Lo que se encontró fue que la mayoría de los estudiantes logró resolver correctamente los problemas financieros del juego. Sin embargo, cuando realizaron un test de habilidad de resolución de problemas financieros, su nivel no puntuó alto. Estos resultados fueron atribuidos a que el videojuego favorecía la resolución de problemas que demandaban conocimientos y habilidades muy específicas, solo para el contexto del juego. La resolución de problemas no respondía a la comprensión de principios tácitos y subyacentes aplicables a contextos tan complejos como los reales. 
Por otro lado, cuando se aborda la flexibilidad del contexto de aplicación desde el nivel de reorientación, se hace referencia a la simulación de contextos complejos en el videojuego donde se demanda del aprendiz/jugador el uso del conjunto de conocimientos y habilidades para resolver problemas con estructuras débilmente definidas, que podrían resolverse de múltiples maneras y está relacionado con diversas áreas de conocimiento (Jonassen, 2011). Otra de las características importantes de este tipo de problemas es el desconocimiento total o parcial de algunos de sus elementos. En este sentido, también hay metas desconocidas que emergen a lo largo del juego y dinamizan la resolución del problema (Marra, Jonassen, Palmer y Luft, 2014).

De acuerdo con esto, cuando el aprendiz aplica el conjunto de conocimientos y habilidades a contextos complejos tiene la posibilidad de comprender principios o ideas subyacentes del área de conocimiento que el videojuego desarrolla. Esta característica la aborda la investigación realizada por Herrero et al. (2014) donde se plantea que, si el aprendiz puede hacer uso de conocimientos y habilidades libremente, se beneficia la comprensión de nuevos conceptos. De esta forma, cuando deba hacer uso de estos conocimientos y habilidades en contextos diferentes al ofrecido por el videojuego, el aprendiz realizará la adaptación necesaria de las habilidades y conocimientos requeridos para el nuevo contexto.

Por ejemplo, en el estudio de Neulight, Kafai, Kao, Foley y Galas (2007) se buscó, por medio de un videojuego, enseñar a estudiantes de secundaria las características de una enfermedad infecciosa y su contagio. El contexto presentado por el videojuego eran lugares de alta concurrencia humana, una playa y una piscina. Este contexto presentaba en su estructura problemas no definidos, donde podían emerger nueva información y metas, lo que demandaba el uso constante de sus conocimientos en biología para cumplir con el objetivo del juego, que era detectar la propagación de una infección y frenarla. El desempeño de los aprendices en el juego fue bueno y desarrollaron de manera compleja el conocimiento en el contexto ofrecido. Además, al realizar un test de conocimiento aplicado donde se les planteaban contextos diferentes a los del videojuego, obtuvieron igualmente buenos desempeños, evidenciados en mejores explicaciones causales basadas en la biología sobre la manera como una infección se contagia. De esta manera, sus conocimientos y habilidades, aplicados a un contexto complejo como el ofrecido por el videojuego, pudieron ser aplicados a contextos complejos reales.

En resumen, para favorecer la calidad de la interacción a partir de los videojuegos, lograr flexibilidad en los contextos de aplicación y comprender fenómenos complejos es importante tener en cuenta principalmente la presencia de dinamismo en la estructura del videojuego educativo (Martí, 2003). El dinamismo implica: la representación de procesos complejos que se transforman en el tiempo; información que se transforma, y poder interactuar con dichos procesos respondiendo a sus condiciones cambiantes (Martí, 2003; Montes y Ochoa, 2006; Valencia et al., 2017). Este dinamismo será la característica esencial para lograr que el nivel de conocimiento y habilidades del aprendiz pueda complejizarse gradualmente a medida que encuentra retos cambiantes en el desarrollo del videojuego. De esta manera, se podrá construir conocimiento y desarrollar habilidades en el videojuego, posiblemente extrapolables a contextos de la vida cotidiana, objetivo último de un videojuego educativo en ciencia, tecnología, ingeniería y matemáticas.

\section{Componentes metacognitivos}

En relación con los aspectos metacognitivos, se encuentran tres características: la explicitación de las metas de aprendizaje; el espacio para la evaluación dentro del proceso metacognitivo y el andamiaje en el uso de estrategias metacognitivas al jugar. La primera tiene que ver con el nivel conciencia metacognitiva que tiene el jugador en relación con lo que se espera conseguir en términos educativos. 
El espacio para la evaluación responde al momento fuera de la acción del juego para reflexionar sobre la comprensión y el desempeño. Finalmente, el andamiaje en el uso de estrategias metacognitivas al jugar hace alusión al nivel de intencionalidad con la que se busca que el jugador reflexione sobre su proceso de construcción del conocimiento, es decir, la cantidad y calidad del apoyo que provee el experto/ docente/par más avanzado para favorecer el desarrollo de las competencias del jugador (Flavell, 1979; Ritterfeld y Weber, 2006; Torrance, 2007).

\section{Explicitación de las metas de aprendizaje}

Dentro del nivel de explicitación de las metas de aprendizaje, en el nivel de integración, estas son planteadas como elementos implícitos dentro del juego y se espera que el estudiante logre cumplirlas de manera autónoma. Torrance (2007) menciona que, al plantear los objetivos de manera implícita, se puede evidenciar el desempeño real de los estudiantes en situaciones de exploración y resolución de problemas abiertos propios de la ciencia. Por eso en ocasiones no se explicitan los objetivos a los estudiantes, ya que se espera que ellos tengan la capacidad de descubrirlos y cumplirlos por sí mismos. Un ejemplo de esto es la investigación de Anderson y Barnett (2011) en la cual estudiantes de primaria debían jugar el videojuego SuperCharged para así poder aprender varios conceptos de electroestática. Se encontró que los estudiantes eran capaces de ganar el juego y discutir en grupo los pasos utilizados y los conceptos aprendidos. Adicionalmente, se encontró que el conocimiento general sobre el contenido se incrementaba después de jugar SuperCharged.

Sin embargo, los autores afirman que, a pesar de que los estudiantes aprendieron los conceptos físicos, muchos no fueron capaces de comprender las relaciones entre conceptos. Por ejemplo, entendieron qué es un campo magnético y qué son cargas estacionarias, pero no fueron capaces de entender cómo ese campo magnético afecta las cargas estacionarias. Pese a que los objetivos no se planteen de manera explícita a los estudiantes, es posible que se dé un avance en la comprensión conceptual. No obstante, esta comprensión será limitada y probablemente se establezca de manera aislada y parcial.

En el nivel de reorientación es importante que los objetivos de aprendizaje se hagan explícitos antes de comenzar a jugar. Para poder reflexionar sobre si se cumplió el objetivo de la tarea o de la clase, es importante que los estudiantes sean conscientes de lo que se les exige, para que así puedan planear cómo conseguirlo y posteriormente evaluar si lo lograron o no y avanzar en la construcción sistemática de conocimiento. Kim, Park, y Baek (2009) plantean que los alumnos pueden contrastar su plan de juego con los objetivos planteados para reflexionar sobre si ese plan les permitirá cumplir con la meta.

Esto se evidencia en la investigación de Schulze et al. (2015) donde se utilizó un videojuego llamado LandYOU para que estudiantes de secundaria y universidad explorarán variables que influyen en el desarrollo de un país, como calidad del ambiente, educación, producción agrícola, recursos financieros, consumo, entre otras. Los autores resaltan la importancia de explicitar las metas de aprendizaje, que en este caso consistían en "encontrar el equilibrio entre condiciones sociales, ambientales y económicas para así alcanzar sostenibilidad". Teniendo en mente esta meta de aprendizaje, los estudiantes iniciaron su experiencia de juego participando en discusiones y debates grupales.

Los resultados mostraron que, partiendo de estas metas explícitas y de discusiones constantes, los estudiantes fueron capaces no solo de evaluar si se cumplió el objetivo de clase, sino también de debatir sobre la relación entre las variables. Los debates científicos fueron valiosos, al permitir comparar el conocimiento previo, el académico y la experiencia de juego. Si no se hubiera explicitado la meta de aprendizaje previamente, el debate no hubiera sido tan potente, ya que los estudiantes no habrían hecho la reflexión previa durante el juego. 


\section{Espacio para la evaluación en el proceso metacognitivo}

Como segunda categoría del proceso metacognitivo está el espacio para la evaluación. La metacognición tiene un momento en el que se dan el monitoreo y un espacio en el que se despliega la evaluación (Flavell, 1979). El monitoreo hace referencia a la manera como el estudiante va revisando si está cumpliendo o no la meta en tiempo real, mientras que la evaluación es el espacio en el que puede volver a pensar sobre su desempeño y reflexionar sobre su proceso de aprendizaje al terminar la actividad. El nivel de integración de esta categoría se caracteriza por que solo se evidencia el monitoreo en tiempo real, es decir, no hay un espacio, al momento de jugar, donde el aprendiz pueda realizar evaluaciones metaestratégicas o metacognitivas con relación al juego.

En esta dirección, Huffaker y Calvert (2003) realizaron una investigación en la que analizaron el uso de la página KineticCity.com (que contiene juegos y actividades con contenido científico) como soporte para el aprendizaje de las ciencias. Los autores plantean que debido a que el juego está dividido en módulos, el estudiante puede controlar y monitorear su desempeño. A pesar de que este monitoreo resulta importante y favorece la construcción y apropiación del conocimiento, no es tan potente, como podría ser si, al final del juego, se brinda un espacio para la evaluación de desempeños, logros y carencias.

El nivel de reorientación se caracteriza por favorecer un espacio de evaluación independiente del de monitoreo, en el que se pueden reorganizar teorías y evidencias. Este espacio de evaluación permite la integración de la teoría y las evidencias, lo cual se logra mediante tres habilidades metacognitivas. La primera es codificar la teoría y la evidencia por aparte, para luego volver sobre ellas y establecer relaciones modificables; la segunda es ver las teorías como unidades independientes de pensamiento, y la última consiste en reconocer la falseabilidad de las teorías (Kuhn, 2005). Estas habilidades permiten que sea posible contrastar las teorías a la luz de las evidencias, para decidir si son verdaderas o no. A su vez los videojuegos facilitan la presentación de evidencia de manera separada a la teoría y así favorecen una mejor y más rápida reorganización.

Un ejemplo de la importancia de un espacio de evaluación para la reorganización de teorías y evidencia es la investigación hecha por Neulight et al. (2007) mediante el juego Whypox y en discusiones en clase, donde los niños lograron formular y contrastar hipótesis sobre el concepto y características de un virus y posteriormente trasladar estos conocimientos a otros contextos y conectarlos con el funcionamiento real de un virus. Los autores concluyeron que el profesor tuvo un papel crucial en la integración del juego en el salón de clase, al abrir espacios de discusión después de las sesiones de juego en las que se preguntaba por los aspectos técnicos del juego, por cómo afectaba el virus (Whypox) a la comunidad del juego, y por las características de Whypox y sus conexiones con las enfermedades naturales. De esta manera se evidencia que, al permitir y fomentar un espacio para la evaluación, se favoreció la reorganización de ideas, lo que finalmente permitió que los estudiantes aprendieran sobre las enfermedades infecciosas virtuales y enlazarlas con las reales.

\section{Andamiaje en el uso de estrategias metacognitivas}

En el nivel de integración de la tercera categoría: el andamiaje en el uso de estrategias metacognitivas al jugar, se asume que la metacognición ya hace parte del repertorio de los estudiantes y, por lo tanto, las características del videojuego harán que emerja naturalmente durante la interacción. Alfageme y Sánchez (2002) afirman que la mayoría de los videojuegos permiten por sí mismos desarrollar habilidades como resolución de problemas, toma de decisiones, organización, coordinación de información de diferentes fuentes, todas ellas importantes dentro de contextos educativos en ciencia. Adicionalmente, Ritterfeld y Weber (2006) plantean que 
los videojuegos permiten el uso de estrategias metacognitivas, las cuales se despliegan incluso desde el primer momento en el que el niño empieza a interactuar con el videojuego.

Un estudio que parte de este supuesto es el de Ko (2002), que comparó las habilidades de resolución de problemas entre dos grupos de edades 7 y 10 años. El videojuego consistía en encontrar un flamenco en una cuadrícula. Si se estaba próximo a encontrar al ave, las celdas del rededor decían caliente y las celdas lejanas decían frío. Los niños que se guiaron por las palabras para encontrar más fácilmente el ave mostraban estrategias metacognitivas y tenían mayor posibilidad de desarrollar nuevas estrategias en el futuro. No obstante, estas habilidades de regulación son básicas y hacen énfasis en procesos como la memoria y la atención y no propician una reflexión metacognitiva centrada en el proceso de aprendizaje. A pesar de que la resolución de problemas puede favorecer la aparición de elementos metacognitivos, como el monitoreo atencional de la actividad, es necesario el favorecimiento explícito y el andamiaje por parte de un experto para potenciar el desarrollo de todo el repertorio de estrategias más complejas.

En contraste, en el nivel de reorientación se plantea la importancia de brindar estrategias metacognitivas al jugador de manera intencional y explícita a través de la integración del videojuego a entornos educativos más amplios. Kim et al. (2009) afirman que las estrategias metacognitivas deben ser enseñadas, debido a que no se dan de manera espontánea. Además, afirman que el uso de las estrategias debe ser monitoreado por el profesor. Finalmente, plantean que para poder dominar estas habilidades es necesario un espacio que favorezca el aprendizaje, por lo que Morris et al. (2013) proponen que estos espacios deben estar culturalmente anclados y apoyarse en el uso de herramientas culturales, en este caso, los videojuegos.

Un ejemplo de este andamiaje explícito de la metacognición es la investigación de Herrero et al. (2014), la cual buscaba evidenciar las posibilidades educativas del uso de un videojuego como herramienta para el aprendizaje de la ciencia en estudiantes de primaria. Al jugar el videojuego Spore, los niños tenían disponibles libros de textos y apuntes, y al final de las sesiones hacían una discusión grupal liderada por su profesor. La función inicial del docente era explicar la tarea y los objetivos principales, además de fomentar la discusión de grupo para aclarar dudas o conceptos erróneos.

En la investigación se encontró que la combinación de los conceptos, junto con las dinámicas del videojuego y el espacio para desarrollar una discusión metacognitiva con el profesor y los pares, permitían que los estudiantes reflexionaran sobre cómo entendían el juego, las estrategias que utilizaron y si alcanzaron los objetivos de la clase. Adicionalmente, encontraron que Spore permitió a los estudiantes identificar y reflexionar sobre su conocimiento previo sobre la evolución y la selección natural para compararlo con el conocimiento científico. El anclaje del juego al contexto de la clase y, sobre todo, a una reflexión metacognitiva más amplia a partir de discusiones guiadas sobre el tema puede favorecer de manera más profunda el desarrollo de la metacognición, mejor que en los casos en los que solo se espera su aparición espontánea.

En sintesis, para el favorecimiento de los aspectos metacognitivos es de suma importancia hacer explícito cuál es el objetivo de estar jugando y cómo está el jugador desarrollando esta actividad de aprendizaje con el videojuego. Esto se logra con guías externas al videojuego, como el acompañamiento docente a lo largo del proceso y/o material físico, como guías escritas, donde se promueva el autocuestionamiento del aprendiz/jugador con respecto a cómo está funcionando él con respecto a la tarea que le propone el videojuego.

\section{Componentes motivacionales}

Es evidente la experiencia de flujo o compromiso de los niños cuando están jugando sus videojuegos favoritos: parecen disfrutar profundamente 
de la actividad y se ven completamente concentrados en lo que hacen. Están tan concentrados que no se dan cuenta del paso del tiempo, y si los padres o profesores los llaman parecen no escuchar. Una vez terminan la actividad, vuelven a conectarse con el mundo y muestran genuino interés en volver a jugar. Este tipo de experiencias han sido descritas en la literatura sobre motivación como experiencias de flujo (Csíkszentmihályi, 2008) y están asociadas con el disfrute de actividades que se han escogido por el placer de realizarlas. La literatura sobre la relación entre disfrute durante los videojuegos y autonomía ha encontrado que los juegos que se disfrutan más son aquellos escogidos por los niños y que se realizan por el gusto de hacerlos, más que por una recompensa externa (McGonigal, 2011; Przybylski, Ryan, y Rigby, 2009, Ryan, Rygby y Przybylski, 2006).

La motivación intrínseca se incrementa cuando el jugador encuentra dentro del juego más oportunidades para ejercer su autonomía, en términos de escogencia de misiones, maneras de alcanzar los objetivos, creación de personajes y escenarios del juego. A su vez, estas posibilidades se expanden cuando el diseño del videojuego implica una "generación procedural" en donde el medio ambiente del juego lo generan las ideas de los múltiples jugadores que lo han jugado alrededor del mundo, con lo que crean posibilidades ilimitadas de elección (Przybylsky, Rigby y Ryan, 2010).

Esta aproximación se corresponde con el denominado nivel de reorientación. En este nivel se considera la motivación como un estado que emerge dependiendo de la interacción entre múltiples factores: la motivación intrínseca del sujeto para jugar el juego, la retroalimentación ofrecida por el videojuego, el nivel de reto, la naturaleza del contenido, así como el nivel de habilidad real y percibido. Por ejemplo, Shernoff, Hamari y Rowe (2014) estudian los estados motivacionales que emergen en los participantes cuando hacen uso de un videojuego educativo. Esta aproximación es realizada a partir de un muestreo de experiencias que indaga por el compromiso de los participantes en el juego en el momento en que lo están jugando. Las conclusiones del estudio señalan que la motivación es emergente debido a diversas características, tanto del jugador como del videojuego, y a la relación entre ellos.

En contraste, hay autores que sugieren que la motivación de los estudiantes para participar en videojuegos aumenta por algún tipo de recompensas que obtienen al jugar (King, Delfabbro y Griffiths, 2009). Algunos recompensan con dinero simbólico o con objetos que dan la posibilidad de mejorar el aspecto visual del juego o la jugabilidad misma. Otra forma de recompensa simbólica es el uso de frases que animan al jugador o posicionan su desempeño en una escala de valor, además de la elaboración de rankings que comparan el desempeño del jugador con sus pares.

Investigaciones sobre fluidez en el juego y factores asociados con su emergencia señalan que esta se produce en situaciones donde la persona tiene metas claras sobre la tarea que realiza, recibe retroalimentación casi inmediata sobre los procesos que lleva a cabo y hay un balance entre el reto que la tarea propone y las habilidades que posee (Csíkszentmihályi, 2008). Estos mismos factores se han estudiado en la emergencia de las experiencias óptimas durante la participación en videojuegos educativos (Kiili, De Freitas, Arnab y Lainema, 2012; Kiili, Perttula, Lindstedt, Arnab y Suominen, 2014; Morris et al., 2013; Shaffer y Fang, 2015). A continuación, se presentan los resultados más importantes de estos estudios.

\section{Importancia de las metas claras en el diseño de los videojuegos}

Kiili et al. (2012), en un estudio realizado con menores universitarios de Finlandia, encontraron que los estudiantes reportaban mayores niveles de compromiso con la realización de videojuegos cuando sabían exactamente qué hacer para llegar a la meta. Los autores argumentan que la claridad en las metas garantiza que los jugadores puedan concentrarse más fácilmente en las tareas que les 
permitirán resolver el problema que plantea el juego. En este sentido, plantean que un adecuado diseño de videojuegos educativos implica presentar la meta principal del juego desde el principio, divida en submetas, de tal manera que el jugador perciba un adecuado manejo del tiempo y de los procesos, lo que aporta sentimientos de éxito durante el juego. En estudios más recientes, Kiili et al. señalan que el establecimiento de metas, cuando el videojuego está diseñado correctamente, permite al jugador moverse hacia experiencias de flujo: "la claridad y propósito ocurre momento a momento y el jugador está tan concentrado en la tarea que las respuestas a las pistas que proporciona el juego pueden optimizarse" (2014, p. 36). Shaffer y Fang (2015) aclaran, retomando a Csíkszentmihályi y Nakamura (2010), que lo más importante no es la meta principal de la actividad, sino que el videojuego sea lo suficientemente claro para presentar la meta global y los pasos en secuencias de acción, hasta que la meta final sea alcanzada.

\section{Retroalimentación inmediata: factor que promueve fluir durante los videojuegos}

La retroalimentación es un factor clave para promover experiencias óptimas como el fluir y el compromiso durante la realización de videojuegos. El objetivo de la retroalimentación es informar al jugador acerca de sus progresos para llegar a la meta. En la medida en que el jugador obtenga una retroalimentación casi inmediata, no se distraerá y estará más centrado en la tarea. Esta idea es coherente con estudios recientes que sugieren que la retroalimentación en los videojuegos debería darse dentro del juego en forma de andamiaje que toma en cuenta el conocimiento del jugador -a partir de sus desempeños- y da información de forma personalizada (Kiili et al., 2012). Este tipo de retroalimentación se encuentra en videojuegos que hemos ubicado en el nivel de reorientación. Se caracteriza por ser constante en el desarrollo del videojuego. En este sentido, la mayoría de acciones realizadas por el jugador tendrán una respuesta por parte del vi- deojuego, que le informará qué tan acertada fue su decisión y si debe replanteársela en alguna medida. Este tipo de retroalimentación le permite al jugador mantener su compromiso con el videojuego, pues encuentra una respuesta inmediata por parte de él.

Un ejemplo de este tipo de retroalimentación se presenta en el estudio de Schaffer y Fang (2015) donde, por medio de un estudio experimental, se busca identificar la diferencia en la motivación emergente, en estudiantes universitarios, cuando hay presencia y ausencia de retroalimentación inmediata en el progreso de un videojuego. El videojuego consistía en calcular el momento exacto de encuentro de figuras geométricas que recorren caminos perpendiculares de ida y vuelta y, a medida que se lograba el objetivo, el participante accedía a un nivel más complejo. Los resultados obtenidos dan cuenta de que los participantes que obtenían retroalimentación inmediata mientras jugaban lograban estados de flujo motivacional con mayor facilidad que los participantes que no recibían este tipo de retroalimentación, lo que dan cuenta de la importancia de esta característica para lograr el compromiso de un jugador con el videojuego. En síntesis, "la retroalimentación en tiempo real sirve como una promesa al jugador de que la meta es definitivamente alcanzable y provee motivación para seguir jugando" (McGonigal, 2011, p. 21).

Por otro lado, en el nivel de integración, la retroalimentación se obtiene ocasionalmente en momentos predeterminados dentro del videojuego. Los momentos más comunes son el comienzo y el final de niveles, en las fases de un nivel y al completar secuencias complejas de acciones necesarias para poder avanzar en el videojuego. Este tipo de retroalimentación lo encontró el estudio de Papastergiou (2009), donde se hace uso del juego LearnMem para identificar si la motivación de los estudiantes para jugar aumenta después de su uso. El videojuego consiste en la aplicación de conceptos de computación, específicamente en lo referente a memorias de almacenamiento y trabajo informático. 
En el desarrollo del juego, a los jugadores solo se les informaba sobre su desempeño y avance al final de etapas o niveles. En este sentido, el jugador desconocía cómo se estaba desempeñando en el desarrollo del nivel y qué acciones podría reestructurar en busca de mejorar el resultado. Aunque los resultados del estudio muestran un aumento en los niveles de motivación después de haber jugado, es importante tener en cuenta que la retroalimentación que potencia la construcción de conocimiento y el desarrollo de habilidades no debe darse únicamente en algunos momentos del videojuego, sino durante todo el proceso mientras se juega.

\section{Balance entre retos de los videojuegos y habilidades del jugador}

Otro factor importante para que el jugador logre un estado de flujo durante el juego es que este represente un reto alcanzable, que le permita avanzar de nivel (Hamari et al., 2016; Oin, Rau y Salvendy, 2010). Los jugadores, grandes y chicos, reportan que parte de la diversión de los videojuegos es que sean difíciles (Jansz, Avis, y Vosmeer, 2010; Olson, 2010). En un estudio sobre el impacto de los videojuegos en adolescentes, Olson et al. (citados en Olson, 2010) indagaron qué era lo que hacía divertido jugar un juego más de una vez y los niños respondieron que el reto era un factor importante: señalaron que un juego que era fácil de jugar y no requería mucha concentración no era divertido.

Hamari et al. (2016) proponen que el nivel de reto es lo que permite que haya aprendizaje, pues a medida que el jugador se vuelve más habilidoso tiende a perder emoción el juego y para recuperar el nivel de disfrute el jugador debe buscar más retos, y esto a la larga lo hace más experto en el juego y en dominar las habilidades que le demandan. Igualmente, estos autores encuentran que durante el videojuego los niveles de reto estuvieron asociados con el compromiso, la sensación de inmersión y la percepción de aprendizaje.
McGonigal (2011) señala que un buen videojuego siempre se juega al borde del nivel de habilidad, siempre a punto de "fallar", y que cuando se "falla" se siente la necesidad de volver a subir, porque la diversión está en poner la propia habilidad al límite. Sin embargo, es necesario aclarar que el nivel de reto debe estar en balance con el de habilidades, pues un videojuego demasiado retador podría generar ansiedad en lugar de experiencias de flujo, y uno demasiado fácil, llevaría al aburrimiento (Kiili et al., 2012; 2015).

Schulze et al. (2015) estudiaron la motivación en un grupo de estudiantes en relación con un videojuego que tenía como objetivo hacer uso adecuado de recursos naturales. El videojuego en su desarrollo le presentaba al jugador desafíos cada vez más difíciles, entre estos, se encontraban cambios imprevistos en las condiciones climáticas o geológicas de su territorio. Los investigadores concluyeron que la presencia de niveles altos de motivación intrínseca y retos constantes para el jugador favoreció estados de flujo a lo largo del videojuego. Resultados similares han encontrado Hamari y Koivisto (2014) y Hung, Sun y Yu (2015).

Especificamente, Hung et al. (2015) utilizaron un juego de TPCS llamado Motion Math: Hungry Fish para ayudar a niños a aprender a sumar y a restar. Los niños fueron divididos en dos grupos: los que hacían ejercicios de emparejamiento y los que participaban en un videojuego con 18 niveles de dificultad. Los hallazgos mostraron que los niños que se enfrentaron al videojuego con los 18 niveles de dificultad tuvieron experiencias de flujo, mostraron más satisfacción durante el juego y aprendieron más.

\section{Conclusiones}

En conclusión, este texto presenta las diferentes posturas de diversas investigaciones frente a las dimensiones cognitivas, metacognitivas y motivacionales que se deberían tener en cuenta cuando se haga uso de un videojuego en un contexto educativo. Estas posturas dejan ver que existen ten- 
dencias marcadas, principalmente, por el lugar que les dan las investigaciones a los videojuegos, desde su aproximación teórica. Se encuentran investigaciones que conciben el videojuego como una herramienta potencial por el hecho de ser un medio audiovisual que favorece el uso de información y movimiento de información, e investigaciones que ven en el videojuego una herramienta que podría ser potente, en la medida en que se use de una manera adecuada y anclada con el contexto educativo en el que se quiere incluir.

Para finalizar, sería posible, a partir de esta revisión, plantear unas recomendaciones específicas para el diseño de videojuegos educativos en el contexto de la enseñanza de la ciencia, para aprovechar todo su potencial. Las recomendaciones presentadas a continuación parten de la idea según la cual un diseño con características funcionales es deseable, pero es posible ir más allá, explotando las posibilidades de los videojuegos como una herramienta cultural que no se parece a ninguna otra.

- Reconocer la acción de jugar para aprender como un escenario donde median múltiples variables que provienen del videojuego, aprendiz/jugador y la relación entre ellos.

- Dinamizar los componentes del videojuego, con estructuras variables, donde el reto presentado por el videojuego sea cambiante, con varios niveles de dificultad, y favorezca la adaptación de la habilidad del aprendiz/jugador.

- Reconocer la relevancia del andamiaje y del material de acompañamiento externo al que ofrece el videojuego para favorecer la reflexión metacognitiva.

- $\quad$ Los retos cambiantes que demande el videojuego deben ser lo suficientemente exigentes como para que el aprendiz/jugador deba también adaptar constantemente el nivel de sus habilidades.

- El desarrollo del pensamiento científico requiere hacer frente a problemas complejos, multivariados y muchas veces poco estructurados. En este sentido, es necesario que el videojuego presente en su estructura este tipo de problemas.

- $\quad$ Aunque no es el punto central de un videojuego educativo, también es importante tener en cuenta que los elementos de forma y contenido del videojuego creen una realidad perceptualmente llamativa para el aprendiz/jugador.

- $\quad$ El diseño de la narrativa del videojuego debe ser realizada de forma rigurosa buscando favorecer la inmersión del aprendiz/jugador en el juego. Esto, teniendo en cuenta que debe haber coherencia y sinergia entre la realidad simulada por el juego y el objetivo educativo planteado.

\section{Referencias}

Alfageme, B. y Sánchez, P. (2002). Aprendiendo habilidades con videojuegos. Revista Cientifica de Comunicación y Educación, 19. Recuperado de http://mail.quadernsdigitals.net/datos_web/hemeroteca/r_2/ nr_664/a_8927/8927.pdf

Anderson, A., Brunner, C., Culp, K. M., Diamond, J., Lewis, A.y Martin, W. (2009). Using microgenetic methods to investigate problem solving in video games. En W. Huber (presidencia), Breaking new ground: Innovation in games, play, practice and theory. Conferencia llevada a cabo en Digital Games Reaserch association (DIGRA), West London, Reino Unido.

Anderson, J. y Barnett, M. (2011). Using video games to support pre-service elementary teachers learning of basic physics principles. Journal of Science Education and Technology, 20(4), 347-362. DOI: 10.1007/s10956-010-9257-0 
Annetta, L. A. (2008). Video games in education: Why they should be used and how they are being used. Theory into Practice, 47(3), 229-239. Recuperado de https://www.jstor.org/stable/40071547

Barab, S.y Dede, C. (2007). Games and immersive participatory simulations for science education: An emerging type of curricula. Journal of Science Education and Technology, 16(1), 1-3. DOI:10.1007/s10956-007-9043-9

Barzilai, S.y Blau, I. (2014). Scaffolding game-based learning: Impact on learning achievements, perceived learning, and game experiences. Computers \& Education, 70, 65-79. DOI: 10.1016/j.compedu.2013.08.003

Cheng, M., Su, T., Huang, W. y Chen, J. (2014). An educational game for learning human immunology: What do students learn and how do they perceive? British Journal of Educational Technology, 45(5), 820-833. DOI: 10.1111/bjet.12098

Clark, D., Nelson, B., Chang, H., Martínez-Garza, M., Slack, K. y D’Angelo, C. (2011). Exploring Newtonian mechanics in a conceptually-integrated digital game: Comparison of learning and affective outcomes for students in Taiwan and the United States. Computers \& Education, 57(3), 2178-2195. DOI: 10.1016/j.compedu.2011.05.007

Csíkszentmihályi, M. (2008). Flow: the psychology of optimal experience. New York: Harper Perennial.

Flavell, J. H. (1979). Metacognition and cognitive monitoring. A new area of cognitive-developmental inquiry. American Psychologist, 34(10), 906-911. DOI: 10.1037/0003-066X.34.10.906

Halpern, D. F., Millis, K., Graesser, A. C., Butler, H., Forsyth, C. y Cai, Z. (2012). Operation ARA: A computerized learning game that teaches critical thinking and scientific reasoning. Thinking Skills and Creativity, 7, 93-100. DOI:10.1016/j.tsc.2012.03.006

Hamari, J. y Koivisto, J. (2014). Measuring flow in gamification: dispositional flow Scale-2. Computers in Human Behavior, 40,133-143. DOI: 10.1016/j.chb.2014.07.048

Hamari, J., Shernoff, D. J., Rowe, E., Coller, B., Asbell-Clarke, J. y Edwards, T. (2016). Challenging games help students learn: An empirical study on engagement, flow and immersion in game-based learning. Computers in Human Behavior, 54, 170-179. DOI: 10.1016/j.chb.2015.07.045

Herrero, D., Del Castillo, H., Monjelat, N., García-Varela, A., Checa, M. y Gómez, P. (2014). Evolution and natural selection: learning by playing and reflecting. Journal of New Approaches in Educational Research, 3(1), 26. DOI: $10.7821 /$ naer.3.1.26-33

Holbert, N. y Wilensky, U. (2014). Constructible authentic representations: Designing video games that enable players to utilize knowledge developed in-game to reason about science. Technology, Knowledge and Learning 19(1-2), 53-79. DOI: 10.1007/s10758-014-9214-8

Hooper,S.y Rieber, L.P.(1995).Teaching withtechnology. Teaching:Theoryinto Practice, 20(13),154-170.Recuperado de https://www.scirp.org/(S(czeh2tfqyw2orz553k1wor45))/reference/ReferencesPapers.aspx?ReferencelD=1241258

Hou, H.y Li, M. (2014). Evaluating multiple aspects of a digital educational problem-solving-based adventure game. Computers in Human Behavior DOI: 10.1016/j.chb.2013.07.052 
ISSN 0123-1294 | e-ISSN 2027-5358 | Educ.Educ. Vol. 21. No. 3 | Septiembre-diciembre de 2018 | pp. 388-408.

Universidad de La Sabana | Facultad de Educación

Huffaker, D. A. y Calvert, S. L. (2003). The new science of learning: Active learning, metacognition, and transfer of knowledge in e-learning applications. Journal of Educational Computing Research, 29(3), 325-334. DOI: 10.2190/4T89-30W2-DHTM-RTQ2

Hung,C.,Sun,J.C.yYu,P.(2015). The benefits of a challenge:student motivation and flow experience in tabletPC-game-based learning. Interactive Learning Environments, 23(2), 172-190. DOI:10.1080/10494820.2014.997248

Jansz, J., Avis, C. y Vosmeer, M. (2010). Playing The Sims2: An exploration of gender differences in players' motivations and patterns of play. New Media \& Society, 12(2), 235-251. DOI: 10.1177/1461444809342267

Jonassen, D. H. (2011). Design problems for secondary students. National Center for Engineering and Technology Education. Recuperado de https://files.eric.ed.gov/fulltext/ED537388.pdf

Kiili, K., De Freitas, S., Arnab, S. y Lainema, T. (2012). The design principles for flow experience in educational games. Procedia Computer Science, 15, 78-91. DOI: 10.1016/j.procs.2012.10.060

Kiili, K., Perttula, A., Lindstedt, A., Arnab, S.y Suominen, M. (2014). Flow experience in evaluating physically activating collaborative serious games. International Journal of Serious Games, 1(3), 35-49. DOI: 10.17083/ijsg.vii3.23

Kim, B., Park, H. y Baek, Y. (2009). Not just fun, but serious strategies: Using meta-cognitive strategies in game-based learning. Computers and Education, 52(4), 800-810. DOI: 10.1016/j.compedu.2008.12.004

King, D., Delfabbro, P. y Griffiths, M. (2009). The psy chological study of video game players: Methodological challenges and practical advice. International Journal of Mental Health and Addiction, 7(4), 555-562. DOI: 10.1007/s11469-009-9198-0

Ko, S. (2002). An empirical analysis of children's thinking and learning in a computer game context. Educational Psychology, 22(2), 219-233. DOI: 10.1080/01443410120115274

Kuhn, D. (2005). Education for thinking. Londres: Harvard University Press.

Kuhn, D. (2010). Teaching and learning science as argument. Science Education, 94(5), 810-824. DOI: $10.1002 /$ sce.20395

Kuhn, D. (2011). What is scientific thinking and how does it develop? En Handbook of Childhood Cognitive Development (pp. 497-523). Oxford, UK: Wiley-Blackwell.

Kuhn, D. y Pease, M. (2008). What needs to develop in the development of inquiry skills? Cognition and Instruction, 26(4), 512-559. DOI: 10.1080/07370000802391745

Lester, J., Spires, H., Nietfeld, J., Minogue, J., Mott, B.y Lobene, E. (2014). Designing game-based learning environments for elementary science education: A narrative-centered learning perspective. Information Sciences, 264, 4-18. DOI: 10.1016/j.ins.2013.09.005

Marra, R. M., Jonassen, D. H., Palmer, B. y Luft, S. (2014). Why problem-based learning works: Theoretical foundations. Journal on Excellence in College Teaching, 25(3-4), 221-238. Recuperado de http://celt.muohio. edu/ject/issue.php?v=25\&n=3\%20and\%204

Martí, E. (2003). Representar el mundo externamente. Madrid: Aprendizaje. 
McGonigal, J. (2011). Reality is broken: Why games make us better and how they can change the world. Nueva York: Penguin.

Miller, L., Chang, C., Wang, S., Beier, M. y Klisch, Y. (2011). Learning and motivational impacts of a multimedia science game. Computers \& Education, 57(1), 1425-1433. DOI:10.1016/j.compedu.2011.01.016

Montes, J. A. y Ochoa, S. (2006). Apropiación de las tecnologías de la información y comunicación en cursos universitarios. Acta Colombiana de Psicología, 9(2). Recuperado de https://www.redaly c.org/articulo. oa?id=79890209

Montes, J., van Dijk, M., Puche, R. y van Geert, P. (2017). Trajectories of scientific reasoning: A microgenetic study on children's inquiry functioning. Journal for Person-Oriented Research, 3(2), 67-85. DOI: 10.17505/ jpor.2017.07

Morris, B., Croker, S., Zimmerman, C., Gill, D. y Romig, C. (2013). Gaming science: the "Gamification" of scientific thinking. Frontiers in Psychology, 4(607), 1-16. DOI: 10.3389/fpsyg.2013.00607

Neulight, N., Kafai, Y., Kao, L., Foley, B. y Galas, C. (2007). Children's participation in a virtual epidemic in the science classroom: Making connections to natural infectious diseases. Journal of Science Education and Technology, 16(1), 47. DOI: 10.1007/s10956-006-9029-z

Olson, C. K. (2010). Children's motivations for video game play in the context of normal development. Review of General Psychology, 14(2), 180-187. DOI: 10.1037/aoo18984

Papastergiou, M. (2009). Digital game-based learning in high school computer science education: Impact on educational effectiveness and student motivation. Computers \& Education, 52(1), 1-12. DOI: 10.1016/j. compedu.2008.06.004

Przybylski, A. K., Rigby, S. y Ryan, R. (2010). A motivational model of video game engagement. Review of General Psychology 14(2), 154-166. DOI: 10.1037/a0019440

Przybylski, A. K., Ryan, R. M.y Rigby, C. S. (2009). The motivating role of violence in video games. Personality and Social Psychology Bulletin, 35, 243-259. DOI: 10.1177/0146167208327216

Puche-Navarro, R. y Ordóñez, O. (2003). Pensar, experimentar y volver a pensar: Un estudio sobre el niño que experimenta con catapultas. El niño que piensa y vuelve a pensar (pp. 88-177). Cali: Universidad del Valle.

Qin, H., Rau, P.-L. P. y Salvendy, G. (2010). Effects of different scenarios of game difficulty on player immersion. Interacting with Computers, 22, 230-239. DOI: 10.1016/j.intcom.2009.12.004

Quintanal Pérez, F. (2016). Aplicación de herramientas de gamificación en física y química de secundaria. Opción, 32(12), 327-348. Recuperado de https://www.redaly c.org/html/310/31048903016/

Rice, J. (2007). New media resistance: Barriers to implementation of computer video games in the classroom. Journal of Educational Multimedia and Hypermedia, 16(3), 249-261. Recuperado de https://www.learntechlib.org/primary/p/24378/

Ritterfeld, U. y Weber, R. (2006). Video games for entertainment and education. Playing video games: Motives, responses, and consequences (pp. 399-413). Nueva York-Londres: Routledge. 
ISSN 0123-1294 | e-ISSN 2027-5358 | Educ.Educ. Vol. 21. No. 3 | Septiembre-diciembre de 2018 | pp. 388-408.

Universidad de La Sabana | Facultad de Educación

Ryan, R. M., Rigby, C. S. y Przybylski, A. (2006). The motivational pull of video games: A self-determination theory approach. Motivation and Emotion, 30, 347-363. DOI: 10.1007/s11031-006-9051-8

Schulze, J., Martin, R., Finger, A., Henzen, C., Lindner, M., Pietzsch, K., ... Seppelt, R. (2015). Design, implementation and test of a serious online game for exploring complex relationships of sustainable land management and human well-being. Environmental Modelling and Software, 65, 58-66. DOI:10.1016/j.envsoft.2014.11.029

Schaffer, O. y Fang, X. (2015). Finding flow with games: Does Immediate Progress Feedback Cause Flow? Paper presented at the Twenty-first Americas Conference of Information Systems, Puerto Rico.

Shaffer, D., Squire, K., Halverson, R. y Gee, J. (2005). Video games and the future of learning. Phi Delta Kappan, 87(2), 105-111. Recuperado de https://academiccolab.org/resources/gappspaper1.pdf

Shernoff, D., Hamari, J. y Rowe, E. (2014). Measuring flow in educational games and gamified learning environments. En World Conference on Educational Media and Technology 2014, Tampere, Finlandía (pp. 22762281). Association for the Advancement of Computing in Education.

Squire, K. (2008). Video game-based learning: An emerging paradigm for instruction. Performance Improvement Quarterly, 21(2), 7-36. Recuperado de https://www.learntechlib.org/p/113514/

Torrance, H. (2007). Assessment as learning? How the use of explicit learning objectives, assessment criteria and feedback in post-secondary education and training can come to dominate learning. Assessment in Education, 14(3), 281-294. DOI: 10.1080/09695940701591867

Valencia-Molina, T., Serna-Collazos, A., Ochoa-Angrino, S., Caicedo-Tamayo, A., Montes-González, J. y Chávez-Vescance, J. (2017). Competencias y estándares TIC desde la dimensión pedagógica: una perspectiva desde los niveles de apropiación de las TIC en la práctica educativa docente. Cali: Pontificia Universidad Javeriana.

Yang, Y. (2012). Building virtual cities, inspiring intelligent citizens: Digital games for developing students' problem solving and learning motivation. Computers and Education, 59(2), 365-377. DOI: 10.1016/j.compedu.2012.01.012

Zimmerman, B. J.y Schunk, D. H. (eds.) (2012). Self-regulated learning and academic achievement: Theory, research, and practice. Nueva York: Springer Science \& Business Media. 